\title{
On Singular and non Singular Rings
}

\author{
Nazar H. Shuker \\ College of Computer Sciences and Mathematics \\ University of Mosul \\ nazarh_2013@yahoo.com \\ Husam Q. Mohammad \\ College of Computer Sciences and Mathematics \\ University of Mosul \\ husam_alsabawi@yahoo.com \\ Shaimaa H. Ahmad \\ College of Computer Sciences and Mathematics \\ University of Mosul \\ Shaymashatim@uomosul.edu.iq \\ Received on:4/10/2005 Accepted on: 20/11/2005
}

ABSTRACT

In this work, we study singular and non singular rings and we give some new basic properties of such rings and its relation with other rings. Finally, we consider rings for which $\mathrm{R} / \mathrm{Y}(\mathrm{R})$ is regular

Key word: singular ring, singular ideal, regular ring

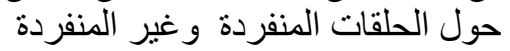

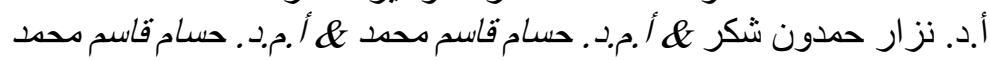

$$
\begin{aligned}
& \text { كلبة علوم الحاسبات والريام فياضيات }
\end{aligned}
$$

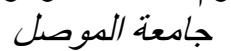

$$
\begin{aligned}
& \text { الكلمات المفتاحية : الحلقات الثاذة , المثاليات المنفردة , الحلقات المنتظمة. }
\end{aligned}
$$

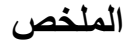

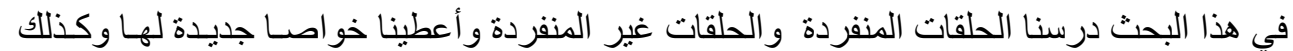

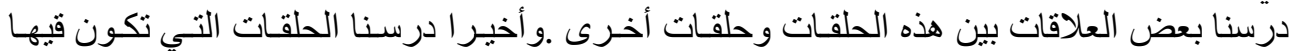

$$
\begin{aligned}
& \text { ملقة منتظمة العلات بين R/Y(R) }
\end{aligned}
$$

\section{Introduction:}

Throughout this paper, $\mathrm{R}$ denotes an associative ring with identity and all modules are unitary right R-modules. Recall that: (1) A ring $\mathrm{R}$ is called right duo-ring if every right ideal is a two-sided ideal in R[5] . (2)A ring $\mathrm{R}$ is called strongly right bounded (briefly $\mathrm{SRB}$ ), if every non-zero right ideal contains a non-zero two sided ideal of $\mathrm{R}$ [1]. (3) A right R-module $\mathrm{M}$ is called a general right principally injective (briefly GP-injective) if for any 
$0 \neq a \in R$, there exists a positive integer $\mathrm{n}$ such that $a^{n} \neq 0$ and any right $\mathrm{R}$-homomorphism of $\mathrm{a}^{\mathrm{n}} \mathrm{R}$ into $\mathrm{M}$ extends to one of $\mathrm{R}$ into $\mathrm{M}[5]$.(4) $\mathrm{A}$ ring $\mathrm{R}$ is called Von Neumann regular ring if for any $a \in R$ there exists $b \in R$ such that $a=a b a$. (5) $r(x), l(x), J(R)$ will stand respectively for the right annihilator of $x$, left annihilator of $x$ and the Jacobson radical of R. (6) A ring $\mathrm{R}$ is said to be reduced if $\mathrm{R}$ contains no non zero nilpotent elements.

\section{Singular Ideal (Basic Properties):}

Recall that the right singular ideal of $R$ is $Y(R)=\{x \in R \mid r(x)$ is essential in $R$ \} . Ring $R$ such that $Y(R)=R$ is called singular, and ring $R$ such that $Y(R)=0$ is called non-singular.

We start this section with the following results:

Lemma 2.1: Let $I$ be a reduced ideal in $R$. Then $a R \cap r(a)=0$ for any element $\mathrm{a}$ in $\mathrm{I}$.

Theorem 2.2: Let $\mathrm{R}$ be a ring. Then $\mathrm{Y}(\mathrm{R}) \cap \mathrm{I}=0$ for any reduced ideal $\mathrm{I}$ in R.

Proof: Let $I$ be a reduced ideal and $Y(R) \cap I \neq 0$. Let $0 \neq a \in Y(R) \cap I$ implies that $\mathrm{r}(\mathrm{a})$ is essential and $\mathrm{a} \in \mathrm{I}$, since $\mathrm{I}$ is a reduced ideal then by Lemma (2.1) we get $a R \cap r(a)=0$. But $r(a)$ is essential therefore $a=0$,a contradiction .Thus $\mathrm{Y}(\mathrm{R}) \cap \mathrm{I}=0$.

Following [4] a ring $\mathrm{R}$ is called zero commutative if for every $\mathrm{a}, \mathrm{b}$ in $\mathrm{R}$ with $\mathrm{a} \cdot \mathrm{b}=0$ implies that $\mathrm{b} . \mathrm{a}=0$.

Theorem2.3: Let $\mathrm{R}$ be zero-commutative ring. Then $\mathrm{Y}(\mathrm{R}) \cap \mathrm{I}=0$ if and only if $I$ is a reduced ideal in $R$.

Proof: Suppose that $Y(R) \cap I=0$, let $0 \neq a \in I$ and $a^{2}=0$ implies that $a \in r(a)$.

We have to prove that $r(a)$ is an essential right ideal of $R$, if not, then there exists a non-zero right ideal $\mathrm{K}$ in $\mathrm{R}$ such that $\mathrm{r}(\mathrm{a}) \cap \mathrm{K}=0$. Then $\mathrm{Ka} \subseteq$ $\mathrm{K} \cap \mathrm{l}(\mathrm{a})$,since R zero-commutative then $\mathrm{l}(\mathrm{a}) \subseteq \mathrm{r}(\mathrm{a})$, so $\mathrm{Ka} \subseteq \mathrm{K} \cap \mathrm{r}(\mathrm{a})=0$ implies that $\mathrm{Ka}=0$, so $\mathrm{K} \subseteq 1(\mathrm{a}) \subseteq \mathrm{r}(\mathrm{a})$ a contradiction .Therefore $\mathrm{r}(\mathrm{a})$ must be essential .

Thus $\mathrm{a} \in \mathrm{Y}(\mathrm{R})$, but $\mathrm{a} \in \mathrm{I}$, so $\mathrm{a} \in \mathrm{Y}(\mathrm{R}) \cap \mathrm{I}=0$. Thus $\mathrm{a}=0$ a contradiction, therefore I must be reduced a ideal in $\mathrm{R}$.

Conversely, follows from Theorem (2.2). 
Theorem 2.4: If $\mathrm{R}$ is $\mathrm{SRB}$ ring, then $\mathrm{N} \subseteq \mathrm{Y}(\mathrm{R})$, where $\mathrm{N}$ is the set of all nilpotent elements of $\mathrm{R}$.

Proof: Let $a^{2}=0$ implies that $a \in r(a)$. If $r(a)$ is not essential there exists a non-zero right ideal $\mathrm{K}$ such that $\mathrm{r}(\mathrm{a}) \oplus \mathrm{K}$ is essential right ideal in $\mathrm{R}$. Since $\mathrm{R}$ is $\mathrm{SRB}$,there exists a non-zero two-sided ideal $\mathrm{I}$ of $\mathrm{R}$ such that $\mathrm{I} \subseteq \mathrm{K}$. Now, $\mathrm{a} I \subseteq \mathrm{a} R \cap I \subseteq \mathrm{r}(\mathrm{a}) \cap \mathrm{K}=0$, hence $\mathrm{I} \subseteq \mathrm{r}(\mathrm{a}) \cap \mathrm{K}=0$.This is a contradiction . Thus $r(a)$ must be an essential right ideal of $R$, implies that $a \in Y(R)$, therefore, $\mathrm{N} \subseteq \mathrm{Y}(\mathrm{R})$.

Corollary 2.5: Let $\mathrm{R}$ be a non-singular SRB ring. Then $\mathrm{R}$ is a reduced ring.

Theorem 2.6: Every right duo-nil ring is singular.

Proof: Let $0 \neq a \in R$ such that $a^{n}=0$, now we have to prove that $r(a)$ is essential. If not, there exists a non-zero right ideal I such that $\mathrm{I} \cap \mathrm{r}(\mathrm{a})=0$ , since $\mathrm{a}^{\mathrm{n}}=0$ implies that $\mathrm{a}^{\mathrm{n}-1} \in \mathrm{r}(\mathrm{a})$ and so $\mathrm{Ia}^{\mathrm{n}-1} \subseteq \mathrm{I} \cap \mathrm{l}(\mathrm{a})$. But $\mathrm{l}(\mathrm{a}) \subseteq \mathrm{r}(\mathrm{a})$ and $I \cap r(a)=0$ therefore $\mathrm{Ia}^{\mathrm{n}-1}=0$.Similarly we get $\mathrm{Ia}=0$ so $\mathrm{I} \subseteq 1(\mathrm{a}) \subseteq \mathrm{r}(\mathrm{a})$ therefore $\mathrm{I}=0$ contradiction so $\mathrm{r}(\mathrm{a})$ is essential .Thus $\mathrm{R}$ is singular .

A ring $\mathrm{R}$ is said to be a right strongly prime if every non-zero ideal I of $\mathrm{R}$ contains a finite subset $\mathrm{F}$ such that $\mathrm{r}(\mathrm{F})=0$. An ideal $\mathrm{P}$ of a ring $\mathrm{R}$ is called strongly prime if the ring $\mathrm{R} / \mathrm{P}$ is strongly prime [3].

Proposition 2.7[1]: Every strongly prime ring $\mathrm{R}$ is nonsingular.

Proof: If $Y(R) \neq 0$, then since $R$ is a right strongly prime, there exists a finite subset $F=\{f 1, \ldots \ldots, \quad \mathrm{fn}\} \subseteq \mathrm{Y}(\mathrm{R})$ such that $r\left(f_{1}\right) \cap r\left(f_{2}\right) \ldots \ldots \cap r\left(f_{n}\right)=r(F)=0$. However $f_{i} \in Y(R)$, so $r\left(f_{i}\right)$ is an essential right ideal of $\mathrm{R}$ for $\mathrm{i}=1, \ldots \ldots \ldots, \mathrm{n}$.

Consequently, $\mathrm{r}(\mathrm{F})$ is an essential right ideal of $\mathrm{R}$, a contradiction. Therefore $\mathrm{Y}(\mathrm{R})=0$.

Let $\mathrm{R}$ be a non-nilpotent finitely generated ring. For every natural number $\mathrm{n}$, the ring $\mathrm{R}^{\mathrm{n}}$ is finitely generated as well. Hence applying Zorn's lemma one can find an ideal $\mathrm{M}$ of $\mathrm{R}$ maximal with respect to $\mathrm{R}^{\mathrm{n}} \not \subset \mathrm{M}$ for $\mathrm{n}=1,2 \ldots \ldots$. The ring $\mathrm{R} / \mathrm{M}$ is strongly prime. Indeed, if $\mathrm{I} / \mathrm{M}$ is a non-zero ideal of $\mathrm{R} / \mathrm{M}$, then the maximality of $\mathrm{M}$ implies that there exists a natural number $\mathrm{n}$, such that $\mathrm{R}^{\mathrm{n}} \subseteq \mathrm{I}$. Let $\mathrm{F}$ be a finite set generating the ring $\mathrm{R}^{\mathrm{n}}$. Then $r_{R / M}(F+M)=r_{R / M}\left(R^{n}+M / M\right)=J / M$ for an ideal $J$ of $R$. If $M \neq J$, then the maximality of $\mathrm{M}$ implies that $\mathrm{R}^{\mathrm{m}} \subseteq \mathrm{J}$ for a natural number $\mathrm{m}$. However, in this case $\mathrm{R}^{\mathrm{n}+\mathrm{m}} \subseteq \mathrm{R}^{\mathrm{n}} \mathrm{J} \subseteq \mathrm{M}$, a contradiction. 
Now from Proposition (2.7) it follows that the ring $\mathrm{R} / \mathrm{M}$ is nonsingular. Taking in particular $\mathrm{R}$ a finitely generated non-nilpotent nil ring one gets that $\mathrm{R} / \mathrm{M}$ is a non singular nil ring . This shows that Theorem (2.6) does not hold for non-commutative nil ring.

$\mathrm{Nam}[5]$ proved the following :

Lemma 2.8: If $a \in \operatorname{Cent}(R)$ with $a=$ ara for some $r \in R$, then there exists $\mathrm{b} \in \operatorname{Cent}(\mathrm{R})$ such that $\mathrm{a}=\mathrm{aba}$ [where $\operatorname{Cent}(\mathrm{R})$ is the Center of $\mathrm{R}$ ] .

Now we give the following main result.

Theorem 2.9: Let $\mathrm{R}$ be a non-singular ring with every simple singular right $\mathrm{R}$-module is GP-injective. Then $\operatorname{Cent}(\mathrm{R})$ is a reduced Von Neumann regular ring.

Proof: First we have to prove that $\operatorname{Cent}(R)$ is reduced. Let $a \neq 0 \in \operatorname{Cent}(R)$ and $a^{2}=0$ implies that $a \in r(a)$.If $r(a)$ is essential then $a \in Y(R)=0$ implies that $a=0$.We are done . If $r(a)$ is not essential, there exists a right ideal $I$ in $R$ such that $\mathrm{r}(\mathrm{a}) \cap \mathrm{I}=0$ and $\mathrm{I} \neq 0$.Then $\mathrm{Ia} \subseteq \mathrm{I} \cap \mathrm{r}(\mathrm{a})[\mathrm{a} \in \operatorname{Cent}(\mathrm{R})]$ but $\mathrm{I} \cap \mathrm{r}(\mathrm{a})=0$ implies that $\mathrm{Ia}=0$ and we get $\mathrm{I} \subseteq \mathrm{I}(\mathrm{a})=\mathrm{r}(\mathrm{a})$ so $\mathrm{I}=0$ contradiction .Therefore $a=0$,so $\operatorname{Cent}(R)$ is a reduced ring . Now we shall show that $a R+r(a)=R$ for any $a \in \operatorname{Cent}(R)$. If not, there exists a maximal right ideal $M$ of $R$ such that $a R+r(a) \subseteq M$, observe that $M$ is an essential right ideal of $R$. If not ,then $M$ is a direct summamd of $R$. So we can write $M=r(e)$ for some $0 \neq e=e^{2} \in R$. Since $\mathrm{a} \in \mathrm{M}$ and $\mathrm{a} \in \operatorname{Cent}(\mathrm{R}), \mathrm{ae}=\mathrm{ea}=0$. Thus $\mathrm{e} \in \mathrm{r}(\mathrm{a}) \subseteq \mathrm{M}=\mathrm{r}(\mathrm{e})$, whence $\mathrm{e}=0$. It is a contradiction. Therefore $\mathrm{M}$ must be an essential right ideal of $\mathrm{R}$. Therefore $\mathrm{R} / \mathrm{M}$ is GP-injective .So there exists a positive integer $\mathrm{n}$ such that any $\mathrm{R}$ homomorphism of $a^{n} R$ into $R / M$ extends to one of $R$ into $R / M$.Let $f: a^{n} R \rightarrow R / M$ be defined by $f\left(a^{n} r\right)=r+M$.Since $a \in \operatorname{Cent}(R)$ and $\operatorname{Cent}(R)$ is reduced, then $\mathrm{f}$ is a well-defined R-homomorphism . Now R/M is GPinjective so there exists $c \in R$ such that $1+M=f\left(a^{n}\right)=c a^{n}+M$. Hence $1-c a^{n} \in M$ and so $1 \in M$. which is a contradiction; therefore, $a R+r(a)=R$ for any $a \in \operatorname{Cent}(R)$ and we have $a=a r a$ for some $r \in R$. Applying Lemma (2.8) Cent(R) is Von Neumann regular ring .

\section{Ring for which $\mathbf{R} / \mathbf{Y}(\mathbf{R})$ Regular}

In this section, we consider rings for which $R / Y(R)$ is regular and we characterize such rings in terms of any ideal $I$ and $Y(R)$.

We shall begin this section with the following result. 
Theorem 3.1: If $R / Y(R)$ is regular ring, then for any reduced ideal $I \in R$ is an idempotent.

Proof: Let $I$ be a reduced ideal in $R$ and $R / Y(R)$ is regular, and let $0 \neq a \in I$, then there exists $r \in R$ such that $a-a r a \in Y(R)$, but $a$-ara $\in I$.

So a-ara $\in \mathrm{I} \cap \mathrm{Y}(\mathrm{R})$ applying Theorem (2.2) we get a-ara=0 implies that $\mathrm{a}=$ ara. Therefore $\mathrm{a} \in \mathrm{I}^{2} \Rightarrow \mathrm{I}=\mathrm{I}^{2}$.

Next we give the following results

Theorem3.2: Let $\mathrm{R}$ be a ring. Then $\mathrm{R} / \mathrm{Y}(\mathrm{R})$ is regular if and only if for any right ideal $\mathrm{I}$ in $\mathrm{R}, \mathrm{I}=\mathrm{I}^{2}+(\mathrm{Y}(\mathrm{R}) \cap \mathrm{I})$.

Proof: Let $R / Y(R)$ be a regular ring and $a \in I$, then there exists $r \in R$ such that $a-a r a \in Y(R)$, but a-ara $\in \mathrm{I}$ so a-ara $\in \mathrm{Y}(\mathrm{R}) \cap \mathrm{I}$.

Now take $\mathrm{k}=\mathrm{a}-\mathrm{ara}$, then $\mathrm{a}=\mathrm{ara}+\mathrm{k}$, but $\operatorname{ara} \in \mathrm{I}^{2}$, therefore $\mathrm{a} \in \mathrm{I}^{2}+(\mathrm{Y}(\mathrm{R}) \cap \mathrm{I})$ implies that $\mathrm{I}^{2}+(\mathrm{Y}(\mathrm{R}) \cap \mathrm{I}) \subseteq \mathrm{I}$ and clearly $\mathrm{I}^{2}+(\mathrm{Y}(\mathrm{R}) \cap \mathrm{I}) \subseteq \mathrm{I}$.Therefore $\mathrm{I}=\mathrm{I}^{2}+(\mathrm{Y}(\mathrm{R}) \cap \mathrm{I})$.

Conversely, let $a \in R$, take $I=a R$ and $a R=(a R)^{2}+(Y(R) \cap a R)$, then there exists $r \in R$ and $x \in Y(R) \cap a R$ such that $a=a r a+x$ implies that a$\operatorname{ara}=x \in Y(R)$, so $a+Y(R)=\operatorname{ara}+Y(R)$.Hence $R / Y(R)$ is regular

Theorem 3.3: Let $R$ be a right duo-ring and $R / Y(R)$ is a regular ring. Then $\mathrm{J}(\mathrm{R}) \subseteq \mathrm{Y}(\mathrm{R})$.

Proof: First we have to prove that $R / Y(R)$ is reduced .Let $a^{2}+Y(R)=Y(R)$ and $a+Y(R) \neq Y(R)$,implies that $a^{2} \in Y(R)$ and $r\left(a^{2}\right)$ is an essential right ideal, let $\mathrm{I} \subseteq \mathrm{r}\left(\mathrm{a}^{2}\right)$ such that $\mathrm{I} \not \subset \mathrm{r}(\mathrm{a})$ so $\mathrm{aI} \subseteq \mathrm{r}(\mathrm{a}) \cap \mathrm{I}$.Therefore $\mathrm{r}(\mathrm{a})$ is an essential ideal in $r\left(a^{2}\right)$ so $r(a)$ is essential in $R$ and $a \in Y(R)$, contradiction.So $R / Y(R)$ is a reduced regular ring and for any $x \in R$ there exists $y \in R$ such that $x-y x^{2} \in Y(R)$. Now let $a \in J(R)$ then, there exists $r \in R$ such that $a-r^{2} \in Y(R)$ and $r\left(a-r^{2}\right)$ is an essential right ideal in $R$. For any $x \in r\left(a-r^{2}\right)$ implies that (1-ra) $a x=0$ and since $a \in J$, there exists $u \in R$ such that $u(1-r a)=1$. So $a x=0$ and implies that $\mathrm{x} \in \mathrm{r}(\mathrm{a})$, therefore $\mathrm{r}\left(\mathrm{a}-\mathrm{ra}^{2}\right) \subseteq \mathrm{r}(\mathrm{a})$. But $\mathrm{r}\left(\mathrm{a}-\mathrm{ra}^{2}\right)$ is essential therefore $\mathrm{r}(\mathrm{a})$ is essential and $\mathrm{a} \in \mathrm{Y}(\mathrm{R})$. So $\mathrm{J}(\mathrm{R}) \subseteq \mathrm{Y}(\mathrm{R})$.

\section{REFERENCES}

[1] Camillo D. M. and H. P. Yu (1994) " Weakly Regular Rings ", Commu. in Algebra 22(10), pp4095-4112 . 
[2] Ferrero M. and E.R.Puczylowski (1998) "The Singular Ideal And Radicals ". Jour. Aust. Math. Soc(series A) 64 ,pp195-209 .

[3] Handelman D.E. and J. Lawrence, (1975) "Strongly Prime Rings ", Trans. Amer. Math. Soc. 211, pp209-233.

[4] Kim N.K. ,S.B.Nam and J.Y. Kim(1999) " On Simple Singular GPInjective Modules ", Commu. in Algebra 27(5), pp2087-2096 .

[5] Nam S.B. (1999) " A Note On Simple Singular GP-Injective Modules " , Kangweon-Kyungki Math. Jour. 7(2), pp215-218. 